\title{
Additional Common Polymorphisms in the PON Gene Cluster Predict PON1 Activity but Not Vascular Disease
}

\author{
Daniel S. Kim, ${ }^{1}$ Amber A. Burt, ${ }^{2}$ Jane E. Ranchalis, ${ }^{2}$ \\ Rebecca J. Richter, ${ }^{2}$ Julieann K. Marshall, ${ }^{2}$ Jason F. Eintracht, ${ }^{3}$ \\ Elisabeth A. Rosenthal, ${ }^{2}$ Clement E. Furlong, ${ }^{1,2}$ and Gail P. Jarvik ${ }^{1,2}$ \\ ${ }^{1}$ Department of Genome Sciences, University of Washington School of Medicine, Seattle, WA 98195, USA \\ ${ }^{2}$ Department of Medicine, Division of Medical Genetics, University of Washington School of Medicine, P.O. Box 357720, \\ Seattle, WA 98195, USA \\ ${ }^{3}$ Department of General Medicine, Virginia Mason Medical Center, Seattle, WA 98101, USA
}

Correspondence should be addressed to Gail P. Jarvik, gjarvik@medicine.washington.edu

Received 1 January 2012; Accepted 14 March 2012

Academic Editor: Bianca Fuhrman

Copyright (C) 2012 Daniel S. Kim et al. This is an open access article distributed under the Creative Commons Attribution License, which permits unrestricted use, distribution, and reproduction in any medium, provided the original work is properly cited.

\begin{abstract}
Background. Paraoxonase 1 (PON1) enzymatic activity has been consistently predictive of cardiovascular disease, while the genotypes at the four functional polymorphisms at PON1 have not. The goal of this study was to identify additional variation at the PON gene cluster that improved prediction of PON1 activity and determine if these variants predict carotid artery disease (CAAD). Methods. We considered 1,328 males in a CAAD cohort. 51 tagging single-nucleotide polymorphisms (tag SNPs) across the PON cluster were evaluated to determine their effects on PON1 activity and CAAD status. Results. Six SNPs (four in PON1 and one each in PON2/3) predicted PON1 arylesterase (AREase) activity, in addition to the four previously known functional SNPs. In total, the 10 SNPs explained $30.1 \%$ of AREase activity, $5 \%$ of which was attributable to the six identified predictive SNPs. We replicate rs 854567 prediction of $2.3 \%$ of AREase variance, the effects of rs3917510, and a PON3 haplotype that includes rs 2375005 . While AREase activity strongly predicted CAAD, none of the 10 SNPs predicting AREase predicted CAAD. Conclusions. This study identifies new genetic variants that predict additional PON1 AREase activity. Identification of SNPs associated with PON1 activity is required when evaluating the many phenotypes associated with genetic variation near PON1.
\end{abstract}

\section{Introduction}

Paraoxonase 1 (PON1) is a liver-produced glycoprotein enzyme bound to the surface of high-density lipoprotein (HDL) whose activity is consistently correlated with atherosclerotic vascular disease and end-organ damage [1$3]$. PON1 is at least partially responsible for the inhibitory effects of HDL on low-density lipoprotein (LDL) peroxidation [4-6] and also has been demonstrated to hydrolyze oxidized lipid or lipid hydroperoxides in LDL [7]. Accordingly, Watson et al. reported that inactivation of PON1 reduced the ability of HDL to inhibit both the oxidation of LDL and the interaction between macrophages and endothelium [6], both likely key factors in the inflammatory changes underlying atherogenesis. It has also been shown that PON1deficient mice cannot neutralize the oxidized LDL lipids and have an increased susceptibility to organophosphate toxicity and coronary heart disease (CHD) $[8,9]$. Finally, PON1 activity appears to play a role in maintaining the endothelialatheroprotective effects of HDL [10].

There are four currently established functional common PON1 single-nucleotide polymorphisms (SNPs) amongst the nearly 200 SNPs in the gene [11]: two missense mutations $\left(P O N 1_{Q 192 R}[\mathrm{rs} 662]\right.$ and $\left.P O N 1_{M 55 L}[\mathrm{rs} 854560]\right)$ and two that alter promoter activity $\left(P O N 1_{-108 C / T}[\mathrm{rs} 705379]\right.$ and $\left.P O N 1_{-162 A / G}[\mathrm{rs} 705381]\right) . P O N 1_{-108 C / T}$ has the largest effect, altering expression likely due to modification of an Sp1 binding site [12]. Rare functional variants have also been identified [13].

While PON1 activity is predictive of vascular disease, studies investigating the role of PON1 SNPs in vascular 
disease have been contradictory [14-18]. A recent metaanalysis of 88 case-control studies by Wang et al. found that $P O N 1_{Q 192}$ was correlated with $\mathrm{CHD}$ [19]. However, removal of smaller studies from the meta-analysis resulted in none of the functional PON1 SNPs having significant association with $\mathrm{CHD}$, thereby replicating the results of past meta-analyses [20-22]. Similarly, our own past investigations have found that while PON1 enzyme levels are predictive of carotid artery disease (CAAD), the genotypes at the four common functional SNPs fail to predict CAAD status [2, 3]. However, studies of CAAD or ischemic strokes are generally more positive for associations with the PON1 functional SNPs $[18,23-26]$ than those for CHD. It should be noted that these studies generally have small sample size and several of such studies reported negative results $[27,28]$.

PON1 has broad substrate specificity and is protective against exposure to toxic organophosphorus insecticides [29]. For biological purposes, PON1 activity is generally measured with regard to the rate of hydrolysis of paraoxon, diazoxon, and phenylacetate (arylesterase activity) [30, 31]. These are termed POase, DZOase, and AREase activities, respectively. AREase enzymatic activity is unaffected by the functional $P O N 1_{Q 192 R}$ polymorphism, thus making it the best reflection of the levels of PON1 protein [32].

PON1 activity has also been linked to a number of other health-related phenotypes in addition to vascular disease and diabetes [33]. For example, PON1 also influences the metabolism of a variety of drugs, including statins, in addition to its aforementioned properties of reducing oxidized LDL and breaking down pesticides [34]. $P O N 1_{\mathrm{Q} 192 \mathrm{R}}$ is a reported determinant of clopidogrel efficacy [35], although this result has not been replicated [36] and remains controversial. PON1 has also been associated with diverse diseases [37]. The $P O N 1_{L 55 M}$ polymorphism has been repeatedly associated with Parkinson's disease [38-40], including a meta-analysis [41], but null results have also been reported [42]. Recent meta-analyses reported the association of PON1 coding SNPs and breast cancer $[43,44]$. Both PON1 activity and genotypes have been associated with age-related macular degeneration [45-51]. PON1 activity is reportedly lower in subjects with systemic lupus erythematosus (SLE) $[48,52-54]$. Finally, diabetes is associated with both reduced PON1 activity and PON1 genotypes [55].

PON1 is one of three paraoxonase gene family members, located in a gene cluster on chromosome 7q21.3-22.1. All of the paraoxonases have antioxidant activity [56]. PON1 and $P O N 3$ share similar functions in association with HDL as described previously; however, $P O N 3$ has lower expression levels [57]. In contrast, PON2 is ubiquitously expressed in human cells [58], particularly in endothelial and aortic smooth muscle cells [59]. PON2 polymorphisms have also been associated with $\mathrm{CHD}[58,60]$. In addition, all three PON gene products have been reported to hydrolyze the quorum sensing factor of Pseudomonas aeruginosa N-3oxododecanoyl homoserine lactone (3OC12-HSL) [61], with PON1 and 2 enzymes specifically being shown in animal knock-out studies to be protective against $p$. aeruginosa infection $[62,63]$.
Carlson et al. previously performed a tagSNP analysis of the PON1, 2, and 3 gene cluster for association with AREase activity and CAAD status in an overlapping, but much smaller, cohort ( $n=500$ versus 1328) [27]. That study found evidence that additional functional SNPs likely exist in PON1, but that the majority of the genetic effect on AREase variation was explained by the four functional SNPs previously described. They did not find evidence for PON2 or PON3 SNPs predicting additional AREase activity.

However, the investigation by Carlson et al. still left a large portion of the variation in PON1 activity unexplained. Thus, the goals of this study are to followup on these previous results and utilize an enlarged cohort and denser tagSNP genotyping to attempt to identify novel common SNPs in the $P O N$ gene cluster that associate with PON1 activity and/or predict CAAD.

\section{Methods}

2.1. Sample. The study population for this analysis consisted of 1,328 samples from the previously described Carotid Lesion Epidemiology And Risk (CLEAR) study $[2,3,64]$. Only Caucasian males were analyzed due to underrepresentation of female and minority samples in this primarily Seattle-Veterans-based cohort. Current smoking status and reported ancestry were obtained by self-report. Ancestry was confirmed using STRUCTURE with three ancestral groups [65]. CAAD status was determined via ultrasound of the internal carotid arteries, with cases defined as having $>50 \%$ stenosis in either artery or a relevant procedure on their carotid arteries in their medical history. Controls had $<15 \%$ stenosis in both arteries. 88 subjects had intermediate stenosis (15-49\%) and were not included for prediction of CAAD, though they were included for prediction of PON1 enzyme activity.

2.2. Genotyping and PON1 Phenotypes. The four known functional PON1 SNPs, $P O N 1_{Q 192 R}, P O N 1_{M 55 L}$, $P O N 1_{-108 C / T}$, and $P O N 1_{-162 A / G}$ and two SNPs identified as potentially predictive by Carlson et al. but not represented on the CVD chip, PON1_-909 (rs854572) and rs3917510 [27], were genotyped using previously described methods $[12,66]$. An additional 86 SNPs in PON1, PON2, and PON3 cluster were genotyped using the Illumina HumanCVD BeadChip (http://www.illumina.com/products/humancvd_whole_genome_genotyping_kits.ilmn). Duplicate genotyping for 34 individuals showed $99.7 \%$ consistency in calls. The PON cluster genotypes were filtered with a minor allele frequency cutoff of $1 \%$ and did not show deviation from HardyWeinberg equilibrium at the $P<10^{-4}$ level. Rs3917564 was also found to be predictive by Carlson et al. and was genotyped by the CVD chip but was not included in the full analysis due to low minor allele frequency $(\mathrm{C} / \mathrm{T}, \mathrm{C}$ allele frequency $=0.008)$.

The PON1 POase, DZOase, and AREase activities were measured by a continuous spectrophotometric assay with lithium heparin plasma, as previously described [66]. AREase activity was measured in duplicate and averaged. 
AREase was utilized as the primary measured outcome of $P O N$ gene cluster variation, due to its closer correlation with protein levels. POase activity is largely determined by the $\mathrm{PON} 1_{\mathrm{Q} 192 \mathrm{R}}$ missense polymorphism, which predicts over $70 \%$ of its variance [2].

2.3. Analysis. LDselect was used to create tagSNPs from the 86 PON1, PON2, and PON3 SNPs genotyped on the Illumina HumanCVD chip [67]. Functional annotation for these SNPs were taken from SNP-Nexus [68]. 51 bins were created, using a linkage disequilibrium (LD) $r^{2}$ threshold of 0.64 . The first 13 of these bins, corresponding to the PON1 gene, had multiple SNPs within them, while the remaining bins consisted of singletons. One SNP from each bin was randomly included in the regression analysis for a total of 51 SNPs in the PON gene cluster. These 51 SNPs did not include the four functional SNPs, which were included in the analysis separately.

We also made an effort to independently replicate SNPs identified as predictive of PON1 activity by Carlson et al. [27]. As our full sample overlaps with that smaller sample, these were tested in a nonoverlapping sample of 523 subjects with complete genotype and phenotype data which were not available at the time of that study.

Regression analysis was done in R (http://www.r-project .org/) using the standard regression tools available. Genotypes were coded using an additive model. Stepwise linear regression was performed, and model comparison was done using Akaike's Information Criterion (AIC) to examine the fit of each model, beginning with a base model that included current smoking status, age, and the genotypes for the four functional PON1 SNPs as covariates [2, 3, 27]. SNPs that are included in the final model increased the ability of the model to predict the dependent variable. Statin drug use can influence PON1 expression, and this appears to be influenced by $P O N 1_{-108}$ genotype [69]. However, statin drug use could not be included as a covariate due to confounding with CAAD status; the preferential use of statins in cases can lead to an erroneous estimation of statin effects on PON1 activity.

\section{Results}

The sample included 1,328 males with a mean age of 67.8 years; $16.5 \%$ of participants reported being current smokers. The subjects included 596 cases and 644 controls considered in the prediction of case status as well as 88 subjects with carotid stenosis between $15-49 \%$ who were considered only for the genotype effects on PON1 activity. Cases had a mean censored (CAAD onset) age of 66.5 years and mean current age at enrollment age of 70.9; controls had a mean current age of 64.6 years. The rates of current smoking and statin use, respectively, were $25.8 \%$ and $64.7 \%$ for cases and $9.6 \%$ and $19.5 \%$ for controls. Descriptions of the 51 tag SNPs for the PON gene cluster are available in Table 1. The AREase activity showed an approximately normal distribution, with a mean of $134 \mathrm{U} / \mathrm{I}$ and standard deviation of 51.8.

A regression model containing functional PON1 SNPs $\left(P O N 1_{Q 192 R}, P O N 1_{M 55 L}, P O N 1_{-108 C / T}\right.$, and $\left.P O N 1_{-162 A / G}\right)$, age, and current smoking status explained $25.2 \%$ of the variance in AREase activity. To explore the possibility of novel SNPs influencing AREase activity, we examined a best-fit model utilizing the stepwise regression including the aforementioned variables plus the 51 tagSNPs. AIC was used to assess whether the additional SNP provided a better fit to the prediction of AREase activity. Only SNPs that added to the predictive power of the best-fit model were kept; others that did not influence the model were discarded. In addition to the four functional SNPs, age, and current smoking, six SNPs were retained in the bestfit model. Together with the original 4 functional SNPs, these additional six SNPs in the PON gene cluster explained $30.1 \%$ of variance in AREase activity (see Table 2). Addition of these SNPs, rs854567, rs2299257, rs2237583, rs2375005, rs3917486, and rs 11768074 serially explained an additional $2.34 \%, 0.85 \%, 0.5 \%, 0.34 \%, 0.58 \%$, and $0.26 \%$ of total variance in PON1 activity. Amongst these six SNPs, four SNPs were in PON1, one was in PON2 (rs2375005), and one was in $P O N 3$ ( $r$ 11768074); all are intronic.

Five of the six SNPs found to predict PON1 activity were the only SNPs in their bin (singletons). The sixth SNP, rs854567, was binned with one other typed SNP, rs2299260, $r^{2}=0.80$. To observe whether it was superior at predicting PON1 AREase activity, we replaced rs854567 with rs2299260 in the complete model of 10 SNPs plus covariates. The model including rs2299260 did not predict additional AREase activity as compared to the model including rs854567, with a total of $30.1 \%$ of AREase variance explained in the full model. Therefore, either SNP or an untyped SNP in LD may be the functional SNP resulting in the association identified.

To address the potential that untyped SNPs are the functional SNPs that underlie the identified AREase associations, the 1000 Genomes database for European ancestry was consulted via SNP-Nexus [68] for these six SNPs. Five of the six SNPs we found to predict PON1 AREase activity were not in strong $\mathrm{LD}\left(r^{2} \geq 0.8\right)$ with other regional SNPs, suggesting that they may be functional. Rs2375005, in contrast, is in strong LD with an additional five SNPs in PON3 $\left(r^{2}=\right.$ 0.901 with intronic rs978903 and synonymous A99A SNP rs1053275; $r^{2}=0.837$ with intronic rs10953146; $r^{2}=0.81$ for intronic rs11970910 and rs117154505) [10].

Prediction of POase activity utilizing these six SNPs that predicted AREase activity (including the base model with age, current smoking status, and the four functional PON1 SNPs) resulted in $84.02 \%$ of POase enzymatic variance explained (see Table 3 ). This compared to $82.74 \%$ of variance explained with the base model with the four functional SNPs, age, and smoking status, with the high percentage of variation explained largely due to the effects of the $P O N_{Q 192 R}$ polymorphism on paraoxon catalytic efficiency. Five of the six SNPs (excluding rs2237583) showed the same directionality of their effects as seen in the AREase analysis, and three had significant effects on POase: rs854567, rs2299257, and rs3917486. When creating a best-fit model that allowed any of the 51 SNPs studied to enter regression in addition to the base model, $84.96 \%$ of POase variance in activity was explained. 
TABLE 1: Characteristics of the 51 SNPs studied in the PON gene cluster.

\begin{tabular}{|c|c|c|c|c|c|}
\hline SNP & Gene & Function $^{\mathrm{a}}$ & $\begin{array}{l}\text { Minor } \\
\text { allele }^{\mathrm{b}}\end{array}$ & $\begin{array}{l}\text { Major } \\
\text { allele }\end{array}$ & MAF \\
\hline rs854549 & PON1 & $3^{\prime}$-downstream & A & $\mathrm{C}$ & 0.337 \\
\hline rs3735590 & PON1 & $3^{\prime}$-UTR & A & G & 0.060 \\
\hline rs3917577 & PON1 & $3^{\prime}$-UTR & G & A & 0.089 \\
\hline rs854552 & PON1 & $3^{\prime}$-UTR & G & A & 0.265 \\
\hline rs3917551 & PON1 & Intronic & A & G & 0.051 \\
\hline rs3917550 & PON1 & Intronic & A & G & 0.137 \\
\hline rs2269829 & PON1 & Intronic & G & A & 0.278 \\
\hline rs3917542 & PON1 & Intronic & A & G & 0.227 \\
\hline rs3917538 & PON1 & Intronic & A & G & 0.236 \\
\hline rs2299257 & PON1 & Intronic & $\mathrm{C}$ & A & 0.391 \\
\hline rs854560 & PON1 & Coding & $\mathrm{T}$ & A & 0.360 \\
\hline rs3917498 & PON1 & Intronic & A & $\mathrm{C}$ & 0.345 \\
\hline rs 28699500 & PON1 & Intronic & G & A & 0.289 \\
\hline rs854561 & PON1 & Intronic & A & G & 0.357 \\
\hline rs854565 & PON1 & Intronic & A & G & 0.294 \\
\hline rs2272365 & PON1 & Intronic & $\mathrm{C}$ & A & 0.154 \\
\hline rs854567 & PON1 & Intronic & A & G & 0.185 \\
\hline rs3917490 & PON1 & Intronic & A & G & 0.490 \\
\hline rs2299261 & PON1 & Intronic & G & A & 0.354 \\
\hline rs854568 & PON1 & Intronic & G & A & 0.219 \\
\hline rs2299262 & PON1 & Intronic & A & G & 0.399 \\
\hline rs854569 & PON1 & Intronic & A & $\mathrm{C}$ & 0.216 \\
\hline rs2237583 & PON1 & Intronic & A & G & 0.284 \\
\hline rs3917486 & PON1 & Intronic & A & G & 0.054 \\
\hline rs3917481 & PON1 & Intronic & A & G & 0.015 \\
\hline rs2237584 & PON1 & Intronic & A & G & 0.058 \\
\hline rs3917478 & PON1 & Intronic & G & A & 0.118 \\
\hline rs3917476 & PON1 & Intronic & A & $\mathrm{C}$ & 0.031 \\
\hline rs854571 & PON1 & $5^{\prime}$-upstream & A & G & 0.289 \\
\hline rs13236941 & PON1 & $5^{\prime}$-upstream & A & G & 0.164 \\
\hline rs13228784 & PON1 & Intronic & G & A & 0.255 \\
\hline rs17883513 & PON1 & Intronic & G & A & 0.032 \\
\hline rs17886762 & PON1 & Intronic & A & G & 0.072 \\
\hline rs17883952 & PON1 & Intronic & A & G & 0.052 \\
\hline rs17884000 & PON3 & Intronic & G & A & 0.202 \\
\hline rs9640632 & PON3 & $3^{\prime}$-UTR & G & A & 0.456 \\
\hline rs 468 & PON3 & Intronic & G & A & 0.066 \\
\hline rs11768074 & PON3 & Intronic & A & G & 0.157 \\
\hline rs10487132 & PON3 & Intronic & G & A & 0.390 \\
\hline rs740264 & PON3 & Intronic & $\mathrm{C}$ & A & 0.254 \\
\hline rs 17884563 & Intergenic & Intergenic & $\mathrm{T}$ & A & 0.109 \\
\hline rs17880030 & Intergenic & Intergenic & A & G & 0.199 \\
\hline rs17881071 & Intergenic & Intergenic & A & G & 0.198 \\
\hline rs2375005 & PON2 & Intronic & $\mathrm{T}$ & A & 0.462 \\
\hline rs 12026 & PON2 & Coding & $\mathrm{C}$ & G & 0.240 \\
\hline rs2299264 & PON2 & Intronic & A & G & 0.241 \\
\hline rs7803148 & PON2 & Intronic & A & G & 0.405 \\
\hline rs2158806 & PON2 & Intronic & $\mathrm{C}$ & A & 0.237 \\
\hline rs2286233 & PON2 & Intronic & A & $\mathrm{T}$ & 0.131 \\
\hline
\end{tabular}


TABle 1: Continued.

\begin{tabular}{|c|c|c|c|c|c|}
\hline SNP & Gene & Function $^{\mathrm{a}}$ & $\begin{array}{l}\text { Minor } \\
\text { allele }^{\mathrm{b}}\end{array}$ & $\begin{array}{l}\text { Major } \\
\text { allele }\end{array}$ & $\mathrm{MAF}^{\mathrm{c}}$ \\
\hline rs10259688 & PON2 & Intronic & $G$ & $\mathrm{~A}$ & 0.179 \\
\hline rs730365 & PON2 & Intronic & A & G & 0.132 \\
\hline
\end{tabular}

Abbreviations: $\mathrm{UTR}=$ untranslated region, $\mathrm{MAF}=$ minor allele frequency, intergenic $=$ located between two gene regions.

${ }^{a}$ SNP functional annotation from SNP-Nexus.

${ }^{\mathrm{b}}$ Major and minor allele annotation from the Illumina HumanCVD Bead Chip.

${ }^{\mathrm{c}}$ Minor allele frequencies calculated from the CLEAR study cohort.

TABLE 2: Best-fit model from stepwise linear regression predicting PON1 AREase activity.

\begin{tabular}{|c|c|c|c|c|c|c|}
\hline Variable & Coefficient $( \pm$ SE $)$ & Gene $^{\mathrm{a}}$ & $\mathrm{MAF}^{\mathrm{b}}$ & $t$-statistic ${ }^{c}$ & $\begin{array}{c}\text { AREase } \\
\text { Variation \% }\end{array}$ & $P$ \\
\hline (Intercept) & $284.09( \pm 13.99)$ & - & - & 20.304 & - & $<2.0 \times 10^{-16}$ \\
\hline$P O N 1_{C-108 T}$ & $-24.82( \pm 2.61)$ & (PON1) & 0.43 & -9.498 & $14.10 \%$ & $<2.0 \times 10^{-16}$ \\
\hline$P O N 1_{G-162 A}$ & $4.61( \pm 4.60)$ & (PON1) & 0.18 & 1.002 & $0.21 \%$ & 0.317 \\
\hline$P O N 1_{Q 192 R}$ & $-22.09( \pm 4.20)$ & PON1 & 0.33 & -5.258 & $1.17 \%$ & $1.8 \times 10^{-7}$ \\
\hline$P O N 1_{M 55 L}$ & $-7.05( \pm 3.64)$ & PON1 & 0.42 & -1.94 & $1.01 \%$ & 0.053 \\
\hline Age & $-1.33( \pm 0.15)$ & - & - & -9.014 & $4.29 \%$ & $<2.0 \times 10^{-16}$ \\
\hline Current smoker & $-28.25( \pm 3.63)$ & - & - & -7.776 & $4.42 \%$ & $1.95 \times 10^{-14}$ \\
\hline rs854567 & $-8.19( \pm 4.77)$ & (PON1) & $\mathrm{A}=0.185$ & -1.719 & $2.34 \%$ & 0.086 \\
\hline rs2299257 & $12.66( \pm 3.57)$ & (PON1) & $C=0.391$ & 3.546 & $0.85 \%$ & $4.11 \times 10^{-4}$ \\
\hline rs 2237583 & $11.36( \pm 3.12)$ & (PON1) & $\mathrm{A}=0.284$ & 3.645 & $0.5 \%$ & $2.82 \times 10^{-4}$ \\
\hline rs2375005 & $-8.32( \pm 2.56)$ & (PON2) & $\mathrm{T}=0.462$ & -3.25 & $0.34 \%$ & 0.001 \\
\hline rs3917486 & $14.91( \pm 4.97)$ & $(P O N 1)$ & $\mathrm{A}=0.054$ & 2.998 & $0.58 \%$ & 0.003 \\
\hline rs11768074 & $8.42( \pm 4.48)$ & (PON3) & $A=0.157$ & 1.878 & $0.26 \%$ & 0.061 \\
\hline
\end{tabular}

$\mathrm{SE}=$ standard error, $\mathrm{MAF}=$ minor allele frequency.

${ }^{a}$ Noncoding SNPs are presented in parentheses, for example, (PON1).

${ }^{\mathrm{b}}$ Minor allele frequencies for the four functional SNPs reported through dbSNP. The remaining minor allele frequencies were calculated via the CLEAR cohort. ${ }^{c} t$-statistics and $P$ values were calculated from the coefficients (from all subjects) and standard errors within the best-fit multivariate model by the glm function in $\mathrm{R}$.

TABle 3: Application of best-fit model for PON1 AREase activity to predict PON1 POase activity.

\begin{tabular}{|c|c|c|c|c|c|c|}
\hline Variable & Coefficient $( \pm$ SE) & Gene $^{\mathrm{a}}$ & $\mathrm{MAF}^{\mathrm{b}}$ & $t$-Statistic ${ }^{c}$ & $\begin{array}{c}\text { POase } \\
\text { Variation \% }\end{array}$ & $P$ \\
\hline (Intercept) & $29.36( \pm 1.17)$ & - & - & 24.986 & - & $<2.0 \times 10^{-16}$ \\
\hline$P O N 1_{C-108 T}$ & $-1.91( \pm 0.22)$ & (PON1) & 0.43 & -8.762 & $11.78 \%$ & $<2.0 \times 10^{-16}$ \\
\hline$P O N 1_{G-162 A}$ & $0.78( \pm 0.39)$ & (PON1) & 0.18 & 2.023 & $3.93 \%$ & 0.043 \\
\hline$P O N 1_{Q 192 R}$ & $9.67( \pm 0.35)$ & PON1 & 0.33 & 27.27 & $65.61 \%$ & $<2.0 \times 10^{-16}$ \\
\hline$P O N 1_{M 55 L}$ & $-1.59( \pm 0.30)$ & PON1 & 0.42 & -5.133 & $0.35 \%$ & $3.5 \times 10^{-7}$ \\
\hline Age & $-0.09( \pm 0.01)$ & - & - & -7.475 & $0.78 \%$ & $1.81 \times 10^{-13}$ \\
\hline Current smoker & $-1.27( \pm 0.31)$ & - & - & -4.155 & $0.31 \%$ & $3.56 \times 10^{-5}$ \\
\hline rs854567 & $-1.69( \pm 0.40)$ & (PON1) & $A=0.185$ & -4.246 & $0.54 \%$ & $2.41 \times 10^{-5}$ \\
\hline rs2299257 & $0.92( \pm 0.30)$ & (PON1) & $C=0.391$ & 3.085 & $0.15 \%$ & 0.002 \\
\hline rs2237583 & $-0.35( \pm 0.26)$ & $($ PON1) & $\mathrm{A}=0.284$ & -1.347 & $0.09 \%$ & 0.179 \\
\hline rs 2375005 & $-0.23( \pm 0.21)$ & (PON2) & $\mathrm{T}=0.462$ & -1.081 & $0.00 \%$ & 0.28 \\
\hline rs3917486 & $2.20( \pm 0.42)$ & $(P O N 1)$ & $\mathrm{A}=0.054$ & 5.271 & $0.47 \%$ & $1.7 \times 10^{-7}$ \\
\hline rs11768074 & $0.42( \pm 0.37)$ & (PON3) & $\mathrm{A}=0.157$ & 1.114 & $0.02 \%$ & 0.266 \\
\hline
\end{tabular}

$\mathrm{SE}=$ standard error, $\mathrm{MAF}=$ minor allele frequency.

${ }^{a}$ Non-coding SNPs are presented in parentheses, for example, (PON1).

${ }^{b}$ Minor allele frequencies for the four functional SNPs reported through dbSNP. The remaining minor allele frequencies were calculated via the CLEAR cohort. ${ }^{c} t$-statistics and $P$ values were calculated from the coefficients (from all subjects) and standard errors within the best-fit multivariate model by the glm function in R. 
Similar application to the prediction of DZOase activity utilizing the six SNPs from the predictive AREase model plus the base model (age, current smoking status, and the four functional PON1 SNPs) resulted in 54.85\% of variance explained (see Table 4). Five of the six SNPs (excluding rs11768074) showed the same directionality of their effects, and 4 had significant effects (rs2299257, rs2237583, rs2375005, and rs3917486). When using the four functional SNPs, age, and sex alone, 50.99\% of DZOase activity was explained. However, when allowing any of the 51 tagSNPs to enter the best-fit model, $55.60 \%$ of DZOase activity was accounted for, suggesting that different SNPs may affect DZOase.

We attempted to replicate SNPs previously identified by Carlson et al. as predicting PON1 activity in a nonoverlapping sample of 523 subjects (Table 5), because significance in an overlapping subset does not constitute replication. The SNPs identified by Carlson et al. were rs854549, rs3917564, rs2269829, rs854566, rs854572, and rs3917510. In our full analysis, rs854566 was tagged by $\operatorname{rs} 854567\left(r^{2}=0.93\right)$, which did enter the full model using the full sample and predicted $2.34 \%$ of AREase activity. Rs2269829 and rs854549 were not predictive of AREase in the full dataset. Rs3917564 was not included in the full model analysis due to minor allele frequency $<0.01$. Rs3917510 and rs854572 $\left(P O N_{-909}\right.$ promoter) were not tagged in the CVD chip analysis and were genotyped separately for the replication analysis. When we considered the independent sample to test the six Carlson SNPs in a linear model predicting AREase, which also included age, current smoking status, and the four functional PON1 SNPs, two of the six Carlson et al. findings were replicated. Both rs854566 (Carlson $P=0.014$, current rs854567 $P=1.64 \times 10^{-5}$ ) and rs3917510 (Carlson $P=$ 0.016 , current $P=0.028$ ) were significant in predicting AREase. Moreover, the direction of effect for rs854566 (Carlson coefficient $=-10.6$, current coefficient $=-20.4$ ) and rs3917510 (Carlson coefficient $=16.6$, current coefficient $=14.3$ ) were the same in both analyses.

None of the 10 SNPs identified in our full analyses, including the four previously known and the six newly reported to predict AREase, predicted CAAD status, considering the covariates censored age and current smoking status. Moreover, none of the SNPs had a $P$ value $<0.10$. However, AREase activity, adjusted by age and current smoking status, was highly associated with CAAD status $\left(P=3.62 \times 10^{-6}\right)$, as previously reported in a smaller sample.

\section{Discussion}

Only four PON1 SNPs are well established to affect PON1 activity. These mutations alone account for approximately only $25 \%$ of PON1 AREase activity, leaving a large amount of variation left unexplained. In this study, we utilized denser tagSNP genotyping and a 2.65-fold increased sample size than those previously used in the Carlson et al. study [27] to examine the effects of common variants, demonstrating the presence of additional functional genetic variance within the PON gene cluster. We identified six additional SNPs that predicted AREase activity (rs854567, rs2299257, rs2237583, rs2375005, rs3917486, and rs11768074). All are intronic, with four in PON1 and one each in PON2 (rs2375005) and PON3 (rs11768074). Of these, only rs2375005 was found to be in strong LD with other regional SNPs in the 1000 genomes data, which included a PON3 synonymous SNP (rs1053275). This LD block SNP is also reported to be in weaker LD with a $P O N 1_{-1741 G A}$ promoter region polymorphism (maximum $r^{2}=0.47$ ) [34]. The remaining 5 SNPs associated with AREase may be functional or in weaker LD with a functional site. Rs 854567 alone predicted $2.3 \%$ of the additional variance in AREase; it lies in the first intron of PON1, a common regulatory area.

For the many phenotypes with genetic associations to the PON cluster, knowledge of which SNPs are associated with functional changes is helpful in determining true associations from spurious ones. As discussed above, rs2375005 is in strong LD with an additional five SNPs in PON3 (rs978903, rs1053275, rs10953146, rs11970910, and rs117154505). These include SNPs that have a reported association with sporadic amyotrophic lateral sclerosis [10]. In addition, Riedmaier et al. have demonstrated that a haplotype block including rs2375005 was associated with atorvastatin lactose hydrolysis and increased PON1 mRNA expression in liver tissue [34]. Our results validate the presence of a functional SNP in this haplotype block.

In comparing these results to the six SNPs identified by Carlson et al, we replicate the effects of two SNPs, rs85466 and rs3917510, while failing to replicate four (Table 5) in nonoverlapping data. Rs854566 was represented in our analyses by the tagSNP, $\operatorname{rs} 854567\left(r^{2}=0.93\right)$. In contrast, the effects of rs854549, rs854572, rs3917564, and rs2269829 are not replicated here. Rs854572 is $5^{\prime}$ SNP PON1-909C/G; while it has been associated with AREase level, smaller studies suggested that all of its activity was attributable to LD with the four functional SNPs [66]. The Carlson et al. paper suggested that this site may have independent activity, but we find no additional effects of this site, in an independent sample of 523 subjects. In sum, our current study confirms both the effects of rs854566 or its bin-mate rs 854567 , predicting $2.3 \%$ of AREase activity and the effects of rs3917510, while also identifying five additional tagSNPs that accounted for approximately $2.7 \%$ of PON1 AREase activity that were not accounted for by Carlson et al.

The finding of PON2 and PON3 SNPs (rs2375005 and rs11768074, resp.) predicting PON1 AREase activity is intriguing. The $P O N$ genes are in a cluster and arranged in order from the centromere as PON1, PON3, and PON2. Each is transcribed in the same direction, toward the centromere. Therefore, variants in the PON2 or PON3 genes lie $5^{\prime}$ to PON1. Rs2375005 is in the sixth of eight PON2 introns. Rs11768074 is in the last PON3 intron. Neither PON2 nor PON3 has intrinsic AREase activity [70], suggesting that these SNPs tag effects on PON1. As noted above, SNPs in the PON3 rs2375005 haplotype block have been described to affect PON1 mRNA level [34], thus the effects of these SNPs, or SNPs in LD with them, may regulate PON1 expression.

Recent research in a cohort investigating SLE has linked rs17884563 and rs740264 in the PON3 region [53] and 
TABLE 4: Application of best-fit model for PON1 AREase activity to predict PON1 DZOase activity.

\begin{tabular}{|c|c|c|c|c|c|c|}
\hline Variable & Coefficient ( \pm SE) & Gene $^{\mathrm{a}}$ & $\mathrm{MAF}^{\mathrm{b}}$ & $t$-statistic ${ }^{c}$ & $\begin{array}{c}\text { DZOase } \\
\text { Activity \% }\end{array}$ & $P$ \\
\hline (Intercept) & $154.26( \pm 4.24)$ & - & - & 36.365 & - & $<2.0 \times 10^{-16}$ \\
\hline$P O N 1_{C-108 T}$ & $-8.69( \pm 0.79)$ & (PON1) & 0.43 & -11.054 & $12.82 \%$ & $<2.0 \times 10^{-16}$ \\
\hline$P O N 1_{G-162 A}$ & $5.03( \pm 1.40)$ & (PON1) & 0.18 & 3.597 & $5.10 \%$ & $3.4 \times 10^{-4}$ \\
\hline$P O N 1_{Q 192 R}$ & $-20.41( \pm 1.28)$ & PON1 & 0.33 & -15.944 & $23.71 \%$ & $<2.0 \times 10^{-16}$ \\
\hline$P O N 1_{M 55 L}$ & $-5.03( \pm 1.12)$ & PON1 & 0.42 & -4.498 & $3.39 \%$ & $7.75 \times 10^{-6}$ \\
\hline Age & $-0.44( \pm 0.44)$ & - & - & -9.797 & $4.21 \%$ & $<2.0 \times 10^{-16}$ \\
\hline Current Smoker & $-5.65( \pm 1.11)$ & - & - & -5.103 & $1.28 \%$ & $4.08 \times 10^{-7}$ \\
\hline rs854567 & $-2.26( \pm 1.44)$ & (PON1) & $A=0.185$ & -1.566 & $1.74 \%$ & 0.118 \\
\hline rs2299257 & $4.03( \pm 1.08)$ & $(P O N 1)$ & $C=0.391$ & 3.73 & $0.70 \%$ & $2.03 \times 10^{-4}$ \\
\hline rs 2237583 & $4.69( \pm 0.95)$ & $(P O N 1)$ & $\mathrm{A}=0.284$ & 4.956 & $0.92 \%$ & $8.57 \times 10^{-7}$ \\
\hline rs 2375005 & $-1.73( \pm 0.76)$ & (PON2) & $\mathrm{T}=0.462$ & -2.258 & $0.22 \%$ & 0.024 \\
\hline rs3917486 & $3.42( \pm 1.51)$ & $(P O N 1)$ & $A=0.054$ & 2.275 & $0.27 \%$ & 0.023 \\
\hline rs11768074 & $-0.64( \pm 1.36)$ & $(P O N 3)$ & $A=0.157$ & -0.466 & $0.01 \%$ & 0.641 \\
\hline
\end{tabular}

$\mathrm{SE}=$ standard error, MAF $=$ minor allele frequency.

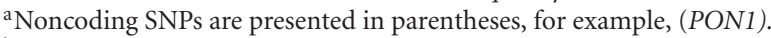

${ }^{b}$ Minor allele frequencies for the four functional SNPs reported through dbSNP. The remaining minor allele frequencies were calculated via the CLEAR cohort.

${ }^{\mathrm{c}} t$-statistics and $P$ values were calculated from the coefficients (from all subjects) and standard errors within the best-fit multivariate model by the glm function in R.

TABLE 5: Comparison of SNPs found significant in prior Carlson et al. ${ }^{\mathrm{a}}$ study with current, non-overlapping sample.

\begin{tabular}{|c|c|c|c|c|c|c|c|}
\hline SNP & $\begin{array}{l}\text { Seattle SNP } \\
\text { annotation }\end{array}$ & $\begin{array}{c}\text { Carlson } \\
\text { coefficient }( \pm \text { SE })\end{array}$ & $\begin{array}{c}\text { Carlson } \\
t \text {-Statistic } \\
\end{array}$ & $\begin{array}{c}\text { Carlson } \\
P^{c}\end{array}$ & $\begin{array}{c}\text { Current } \\
\text { coefficient } \\
( \pm \mathrm{SE})^{\mathrm{d}}\end{array}$ & $\begin{array}{c}\text { Current } \\
t \text {-Statistic }{ }^{b}\end{array}$ & $\begin{array}{c}\text { Current } \\
P^{\mathrm{c}}\end{array}$ \\
\hline rs $854566^{\mathrm{e}}$ & $P O N 1_{6842}$ & $-10.6( \pm 4.3)$ & -2.480 & 0.014 & $-20.4( \pm 4.68)$ & -4.353 & $\begin{array}{l}1.64 \times \\
10-5\end{array}$ \\
\hline rs3917510 & $P O N 1_{12471}$ & $16.6( \pm 6.9)$ & 2.424 & 0.016 & $14.3( \pm 6.48)$ & 2.208 & 0.028 \\
\hline rs2269829 & $P O N 1_{19470}$ & $-16.5( \pm 10.8)$ & -1.520 & 0.129 & $13.6( \pm 21.82)$ & 0.625 & 0.533 \\
\hline rs3917564 & $P O N 1_{23887}$ & $-39.0( \pm 18.1)$ & -2.153 & 0.032 & $15.0( \pm 26.67)$ & 0.564 & 0.573 \\
\hline rs854549 & $P O N 1_{29021}$ & $9.2( \pm 4.5)$ & 2.051 & 0.041 & $-1.3( \pm 4.90)$ & -0.260 & 0.795 \\
\hline rs854572 & $\mathrm{PON}_{895}$ & $13.0( \pm 4.9)$ & 2.677 & 0.008 & $-0.28( \pm 4.97)$ & -0.056 & 0.955 \\
\hline
\end{tabular}

$\mathrm{SE}=$ standard error.

${ }^{a}$ Carlson et al. study $n=500$ European male subjects [27].

$\mathrm{b}_{t}$-Statistics and $P$-values were calculated from the coefficients from each subgroup (Carlson $n=500$, current study $n=523$ ) and standard errors within the best-fit multivariate model by the glm function in R.

${ }^{\mathrm{c} B o t h}$ Carlson and current study utilized a linear regression model adjusting for age, current smoking status, and the four functional PON1 SNPs.

${ }^{\mathrm{d}}$ Current study subset of 523 European male subjects not considered by Carlson et al.

${ }^{\mathrm{e}}$ Represented by proxy SNP, rs854457, with LD $r^{2}=0.93$ in the current study.

five PON2 SNPs [52] (rs6954345, rs13306702, rs987539, rs11982486, and rs4729189) with PON1 POase activity [52, 53]. These investigations utilized POase rather than AREase activity [71]; this is not optimal, as the $P O N 1_{O 192 R}$ activity accounts for most POase activity. Of the PON3 SNPs found to predict POase activity [53], both rs17884563 (intergenic between PON2 and PON3 in our annotation) and rs740264 were directly genotyped and included in our regression model for PON1 AREase activity but were not predictive. When applying rs17884563 or rs740264 to POase activity, which the aforementioned investigators used as their PON1 phenotype, neither was predictive of POase activity. For the PON2 SNPs predictive of POase in the SLE cohort, all five were represented by tag SNPs $\left(r^{2}>0.6\right)$, but only rs2375005 $\left(r^{2}=1\right.$ with rs987539) was predictive of PON1 AREase activity. Interestingly, none of these five PON2 SNPs predict POase in our data, including rs2375005 $(P=0.28)$. The differences in PON2 and PON3 SNP associations between our data and the SLE cohort may reflect differences in cohort selection criteria (older male vascular disease versus younger female SLE, cases and controls) or sizes (1,322 in our data versus 922 in the SLE data).

Application of the six SNPs from the AREase best-fit model to predicting POase and DZOase activity resulted in the prediction of $98.89 \%$ and $98.65 \%$ of enzymatic activity predicted by models, where all 51 SNPs were allowed to enter. Three of these six SNPs, all in PON1, also predict both PON1 POase and DZOase activities. While it is clear why coding SNPs would differentially influence the PON1 degradation of these three substrates, it is less clear why regulatory variants 
would. Further investigation is required to determine if and how these noncoding SNPs differentially influence PON1's multiple activities at the genomic, molecular, or cellular level.

None of the six new SNPs that predicted AREase activity were predictive of CAAD. In addition, none of the four functional PON1 SNPs were predictive of CAAD, which is consistent with past findings with smaller sample sizes in this cohort $[2,3,27]$. Important sources of variance in AREase activity that are not captured by these genotypes or the covariates of age and current smoking must account for the strong association between this activity and CAAD. Possible sources of AREase variation include rare regional variants, regional gene regulation not captured by genotyping (such as methylation), variation in genes outside the PON cluster, nongenetic factors including statin drug use [72] and diet $[73,74]$, as recently reviewed [75], as well as interactions among these. Evidence of interactions includes the report of the association of PON1 genotype and CHD only in subjects with diabetes [76]. These results emphasize the importance of researching the correlation of PON1 and cardiovascular disease more broadly by utilizing "PON status," taking into account both the genotype of PON1 SNPs and the plasma activity $[11,77]$, as well as investigating factors which affect the specific activity of PON1. PON1 has been suggested as a drug target for vascular and other diseases, thus a clear understanding of its role in disease is crucial [78].

Some limitations of this study must be considered. First, the study was comprised entirely of males of European descent, thereby limiting the generalizations that can be drawn from these findings. Second, this investigation considered only SNPs from the PON gene cluster. Variation in other genes may influence PON1 activity [79]. For example, peroxisome proliferator-activated receptor gamma (PPARG) activates PON1 expression in hepatocytes [80], leading to the possibility that variation in the PPARG gene could alter levels of PON1 protein. However, the larger size of this study and the denser tagSNPing of the PON cluster, relative to the earlier Carlson et al. work [27], allowed us to detect novel genetic variation that predicts PON1 AREase activity.

In conclusion, our analysis of the PON gene cluster identifies six additional common genetic variants that predict AREase activity: four are novel, predicting 2.4\% of AREase activity and two replicate past findings. The replicated SNPs include rs854567, which tags $2.3 \%$ of AREase variance, rs3917510, and rs2375005, which tags $0.3 \%$ of AREase variance. We do not identify additional effects of the PON1-909 polymorphism. Future studies to quantify the role of rare genetic variation and variation outside the $P O N$ cluster on PON1 activity will be important. Finally, the continued lack of an association between PON1, 2, or 3 genetic variants and CAAD, while PON1 activity is highly predictive, underscores the importance of utilizing PON status in future studies investigating the link between PON1 and vascular or other disease.

\section{Abbreviations}

AIC: Akaike's Information Criterion

CAAD: Carotid artery disease
CHD: Coronary heart disease

CLEAR: Carotid Lesion Epidemiology and Risk cohort

DZOase: Diazoxon enzymatic hydrolysis

POase: Paraoxon enzymatic hydrolysis

PON: Paraoxonase

tagSNP: Tagging single-nucleotide polymorphism.

\section{Conflict of Interests}

The authors declare that they have no conflict of interests.

\section{Acknowledgments}

The authors would like to thank the study participants. They would also like to thank the following people for their technical assistance: Tamara Bacus, Edward Boyko, Julieann Marshall, Laura McKinstry, Karen Nakayama, Jane Ranchalis, and Jeff Rodenbaugh. This paper was funded by NIH RO1 HL67406 and a State of Washington Life Sciences Discovery Award to the Northwest Institute of Genetic Medicine. D. S. Kim is supported by a Sarnoff Cardiovascular Research Fellowship for Medical Students Award. This work utilized resources of SeattleSNPs; NHLBI Program for Genomic Applications, SeattleSNPs, Seattle, WA (http://pga.gs.washington.edu/). Past work in this cohort was supported in part by resources from the VA Puget Sound Health Care System, Seattle, Washington, including the Veteran Affairs Epidemiology Research and Information Center Program (Award CSP 701S).

\section{References}

[1] M. I. Mackness, B. Mackness, and P. N. Durrington, "Paraoxonase and coronary heart disease," Atherosclerosis Supplements, vol. 3, no. 4, pp. 49-55, 2002.

[2] G. P. Jarvik, L. S. Rozek, V. H. Brophy et al., "Paraoxonase (PON1) phenotype is a better predictor of vascular disease than is PON1192 or PON155 genotype," Arteriosclerosis, Thrombosis, and Vascular Biology, vol. 20, no. 11, pp. 24412447, 2000.

[3] G. P. Jarvik, T. S. Hatsukami, C. Carlson et al., "Paraoxonase activity, but not haplotype utilizing the linkage disequilibrium structure, predicts vascular disease," Arteriosclerosis, Thrombosis, and Vascular Biology, vol. 23, no. 8, pp. 1465-1471, 2003.

[4] M. I. Mackness, S. Arrol, C. Abbott, and P. N. Durrington, "Protection of low-density lipoprotein against oxidative modification by high-density lipoprotein associated paraoxonase," Atherosclerosis, vol. 104, no. 1-2, pp. 129-135, 1993.

[5] A. Graham, D. G. Hassall, S. Rafique, and J. S. Owen, "Evidence for a paraoxonase-independent inhibition of lowdensity lipoprotein oxidation by high-density lipoprotein," Atherosclerosis, vol. 135, no. 2, pp. 193-204, 1997.

[6] A. D. Watson, J. A. Berliner, S. Y. Hama et al., "Protective effect of high density lipoprotein associated paraoxonase. Inhibition of the biological activity of minimally oxidized low density lipoprotein," Journal of Clinical Investigation, vol. 96, no. 6, pp. 2882-2891, 1995.

[7] H. Cao, A. Girard-Globa, F. Berthezene, and P. Moulin, "Paraoxonase protection of LDL against peroxidation is independent of its esterase activity towards paraoxon and is 
unaffected by the $\mathrm{Q} \rightarrow \mathrm{R}$ genetic polymorphism," Journal of Lipid Research, vol. 40, no. 1, pp. 133-139, 1999.

[8] D. M. Shih, L. Gu, Y. R. Xia et al., "Mice lacking serum paraoxonase are susceptible to organophosphate toxicity and atherosclerosis," Nature, vol. 394, no. 6690, pp. 284-287, 1998.

[9] D. M. Shih, Y. R. Xia, X. P. Wang et al., "Combined serum paraoxonase knockout/apolipoprotein E knockout mice exhibit increased lipoprotein oxidation and atherosclerosis," Journal of Biological Chemistry, vol. 275, no. 23, pp. 1752717535, 2000.

[10] C. Besler, K. Heinrich, L. Rohrer et al., "Mechanisms underlying adverse effects of HDL on eNOS-activating pathways in patients with coronary artery disease," Journal of Clinical Investigation, vol. 121, no. 7, pp. 2693-2708, 2011.

[11] R. J. Richter, G. P. Jarvik, and C. E. Furlong, "Paraoxonase 1 status as a risk factor for disease or exposure," in Paraoxonases in Inflammation, Infection, and Toxicology, S. T. Reddy, Ed., vol. 660, pp. 29-35, Humana Press, 2010.

[12] V. H. Brophy, M. D. Hastings, J. B. Clendenning, R. J. Richter, G. P. Jarvik, and C. E. Furlong, "Polymorphisms in the human paraoxonase (PON1) promoter," Pharmacogenetics, vol. 11, no. 1, pp. 77-84, 2001.

[13] G. P. Jarvik, R. Jampsa, R. J. Richter et al., "Novel paraoxonase (PON1) nonsense and missense mutations predicted by functional genomic assay of PON1 status," Pharmacogenetics, vol. 13, no. 5, pp. 291-295, 2003.

[14] T. Bhattacharyya, S. J. Nicholls, E. J. Topol et al., "Relationship of paraoxonase 1 (PON1) gene polymorphisms and functional activity with systemic oxidative stress and cardiovascular risk," Journal of the American Medical Association, vol. 299, no. 11, pp. 1265-1276, 2008.

[15] M. Serrato and A. J. Marian, "A variant of human paraoxonase/arylesterase (HUMPONA) gene is a risk factor for coronary artery disease," Journal of Clinical Investigation, vol. 96, no. 6, pp. 3005-3008, 1995.

[16] M. Antikainen, S. Murtomäki, M. Syvänne et al., "The Gln-Arg191 polymorphism of the human paraoxonase gene (HUMPONA) is not associated with the risk of coronary artery disease in Finns," Journal of Clinical Investigation, vol. 98, no. 4, pp. 883-885, 1996.

[17] S. M. Herrmann, H. Blanc, O. Poirier et al., "The Gln/Arg polymorphism of human paraoxonase (PON 192) is not related to myocardial infarction in the ECTIM Study," Atherosclerosis, vol. 126, no. 2, pp. 299-303, 1996.

[18] H. Schmidt, R. Schmidt, K. Niederkorn et al., "Paraoxonase PON1 polymorphism Leu-Met54 is associated with carotid atherosclerosis: results of The Austrian Stroke Prevention Study," Stroke, vol. 29, no. 10, pp. 2043-2048, 1998.

[19] M. Wang, X. Lang, L. Zou, S. Huang, and Z. Xu, "Four genetic polymorphisms of paraoxonase gene and risk of coronary heart disease: a meta-analysis based on 88 casecontrol studies," Atherosclerosis, vol. 214, no. 2, pp. 377-385, 2011.

[20] D. A. Lawlor, I. N. M. Day, T. R. Gaunt et al., "The association of the PON1 Q192R polymorphism with coronary heart disease: findings from the British Women's Heart and Health cohort study and a meta-analysis," BMC Genetics, vol. 5, no. 1, arricle 17, 2004.

[21] J. G. Wheeler, B. D. Keavney, H. Watkins, R. Collins, and J. Danesh, "Four paraoxonase gene polymorphisms in 11212 cases of coronary heart disease and 12786 controls: metaanalysis of 43 studies," The Lancet, vol. 363, no. 9410, pp. 689$695,2004$.
[22] K. E. Lohmueller, C. L. Pearce, M. Pike, E. S. Lander, and J. N. Hirschhorn, "Meta-analysis of genetic association studies supports a contribution of common variants to susceptibility to common disease," Nature Genetics, vol. 33, no. 2, pp. 177$182,2003$.

[23] B. Voetsch, K. S. Benke, B. P. Damasceno, L. H. Siqueira, and J. Loscalzo, "Paraoxonase $192 \mathrm{Gln} \rightarrow$ Arg polymorphism: an independent risk factor for nonfatal arterial ischemic stroke among young adults," Stroke, vol. 33, no. 6, pp. 1459-1464, 2002.

[24] B. Voetsch, K. S. Benke, C. I. Panhuysen, B. P. Damasceno, and J. Loscalzo, "The combined effect of paraoxonase promoter and coding region polymorphisms on the risk of arterial ischemic stroke among young adults," Archives of Neurology, vol. 61, no. 3, pp. 351-356, 2004.

[25] R. Schmidt, H. Schmidt, F. Fazekas et al., "MRI cerebral white matter lesions and paraoxonase PON1 polymorphisms: three-year follow-up of the Austrian stroke prevention study," Arteriosclerosis, Thrombosis, and Vascular Biology, vol. 20, no. 7, pp. 1811-1816, 2000.

[26] H. Markus, Z. Kapozsta, R. Ditrich et al., "Increased common carotid intima-media thickness in UK African Caribbeans and its relation to chronic inflammation and vascular candidate gene polymorphisms," Stroke, vol. 32, no. 11, pp. 2465-2471, 2001.

[27] C. S. Carlson, P. J. Heagerty, T. S. Hatsukami et al., “TagSNP analyses of the PON gene cluster: effects on PON1 activity, LDL oxidative susceptibility, and vascular disease," Journal of Lipid Research, vol. 47, no. 5, pp. 1014-1024, 2006.

[28] E. Topic, A. Timundic, M. Ttefanovic et al., "Polymorphism of Apoprotein E (APOE), methylenetetrahydrofolate reductase (MTHFR) and paraoxonase (PON1) genes in patients with cerebrovascular disease," Clinical Chemistry and Laboratory Medicine, vol. 39, pp. 346-350, 2001.

[29] L. G. Costa, B. E. McDonald, S. D. Murphy et al., "Serum paraoxonase and its influence on paraoxon and chlorpyrifosoxon toxicity in rats," Toxicology and Applied Pharmacology, vol. 103, no. 1, pp. 66-76, 1990.

[30] R. J. Richter, G. P. Jarvik, and C. E. Furlong, "Determination of paraoxonase 1 status without the use of toxic organophosphate substrates," Circulation, vol. 1, no. 2, pp. 147-152, 2008.

[31] R. J. Richter, G. P. Jarvik, and C. E. Furlong, "Paraoxonase 1 (PON1) status and substrate hydrolysis," Toxicology and Applied Pharmacology, vol. 235, no. 1, pp. 1-9, 2009.

[32] C. E. Furlong, N. Holland, R. J. Richter, A. Bradman, A. Ho, and B. Eskenazi, "PON1 status of farmworker mothers and children as a predictor of organophosphate sensitivity," Pharmacogenetics and Genomics, vol. 16, no. 3, pp. 183-190, 2006.

[33] R. W. James, "A long and winding road: defining the biological role and clinical importance of paraoxonases," Clinical Chemistry and Laboratory Medicine, vol. 44, no. 9, pp. 1052-1059, 2006.

[34] S. Riedmaier, K. Klein, S. Winter, U. Hofmann, M. Schwab, and U. Zanger, "Paraoxonase (PON1 and PON3) polymorphisms: impact on liver expression and atorvastatin-lactone hydrolysis," Frontiers in Pharmacology, vol. 2, p. 41, 2011.

[35] H. J. Bouman, E. Schömig, J. W. Van Werkum et al., "Paraoxonase-1 is a major determinant of clopidogrel efficacy," Nature Medicine, vol. 17, no. 1, pp. 110-116, 2011.

[36] J. P. Lewis, A. S. Fisch, K. Ryan et al., "Paraoxonase 1 (PON1) gene variants are not associated with clopidogrel response," Clinical Pharmacology and Therapeutics, vol. 90, no. 4, pp. 568-574, 2011. 
[37] B. Mackness, M. I. Mackness, M. Aviram, and G. Paragh, Paraoxonases: Their Role in Disease Development and Xenobiotic Metabolism (Proteins and Cell Regulation), Springer, 2007.

[38] A. Carmine, S. Buervenich, O. Sydow, M. Anvret, and L. Olson, "Further evidence for an association of the paraoxonase 1 (PON1) met-54 allele with Parkinson's disease," Movement Disorders, vol. 17, no. 4, pp. 764-766, 2002.

[39] I. Kondo and M. Yamamoto, "Genetic polymorphism of paraoxonase 1 (PON1) and susceptibility to Parkinson's disease," Brain Research, vol. 806, no. 2, pp. 271-273, 1998.

[40] S. N. Akhmedova, A. K. Yakimovsky, and E. I. Schwartz, "Paraoxonase 1 Met-Leu 54 polymorphism is associated with Parkinson's disease," Journal of the Neurological Sciences, vol. 184, no. 2, pp. 179-182, 2001.

[41] E. Zintzaras and G. M. Hadjigeorgiou, "Association of paraoxonase 1 gene polymorphisms with risk of Parkinson's disease: a meta-analysis," Journal of Human Genetics, vol. 49, no. 9, pp. 474-481, 2004.

[42] S. N. Kelada, P. Costa-Mallen, H. Checkoway et al., "Paraoxonase 1 promoter and coding region polymorphisms in Parkinson's disease," Journal of Neurology Neurosurgery and Psychiatry, vol. 74, no. 4, pp. 546-547, 2003.

[43] S. Mostafa, "Paraoxonase 1 genetic polymorphisms and susceptibility to breast cancer: a meta-analysis," Cancer Epidemiology, vol. 36, no. 2, pp. e101-e103, 2012.

[44] C. Liu and L. Liu, "Polymorphisms in three obesity-related genes (LEP, LEPR, and PON1) and breast cancer risk: a metaanalysis," Tumor Biology, vol. 32, no. 6, pp. 1233-1240, 2011.

[45] A. Javadzadeh, A. Ghorbanihaghjo, E. Bahreini, N. Rashtchizadeh, H. Argani, and S. Alizadeh, "Serum paraoxonase phenotype distribution in exudative age-related macular degeneration and its relationship to homocysteine and oxidized low-density lipoprotein," Retina, vol. 32, no. 4, pp. 658666, 2012.

[46] M. Briõn, M. Sanchez-Salorio, M. Cortõn et al., "Genetic association study of age-related macular degeneration in the Spanish population," Acta Ophthalmologica, vol. 89, no. 1, pp. e12-e22, 2011.

[47] G. J. T. Pauer, G. M. Sturgill, N. S. Peachey, and S. A. Hagstrom, "Protective effect of paraoxonase 1 gene variant Gln192Arg in age-related macular degeneration," American Journal of Ophthalmology, vol. 149, no. 3, pp. 513-522, 2010.

[48] O. Ates, S. Azzi, H. H. Alp et al., "Decreased serum paraoxonase 1 activity and increased serum homocysteine and malondialdehyde levels in Age-related macular degeneration," Tohoku Journal of Experimental Medicine, vol. 217, no. 1, pp. 17-22, 2009.

[49] H. Esfandiary, U. Chakravarthy, C. Patterson, I. Young, and A. E. Hughes, "Association study of detoxification genes in age related macular degeneration," British Journal of Ophthalmology, vol. 89, no. 4, pp. 470-474, 2005.

[50] G. Baskol, S. Karakucuk, A. O. Oner et al., "Serum paraoxonase 1 activity and lipid peroxidation levels in patients with age-related macular degeneration," Ophthalmologica, vol. 220, no. 1, pp. 12-16, 2005.

[51] P. N. Baird, D. Chu, E. Guida, H. T. V. Vu, and R. Guymer, "Association of the M55L and Q192R paraoxonase gene polymorphisms with age-related macular degeneration," American Journal of Ophthalmology, vol. 138, no. 4, pp. 665-666, 2004.

[52] S. Dasgupta, F. Y. Demirci, A. S. Dressen et al., "Association analysis of PON2 genetic variants with serum paraoxonase activity and systemic lupus erythematosus," BMC Medical Genetics, vol. 12, article 7, no. 1, 2011.
[53] D. K. Sanghera, S. Manzi, R. L. Minster et al., "Genetic variation in the paraoxonase-3 (PON3) gene is associated with serum PON1 activity," Annals of Human Genetics, vol. 72, no. 1, pp. 72-81, 2008.

[54] E. Kiss, I. Seres, T. Tarr, Z. Kocsis, G. Szegedi, and G. Paragh, "Reduced paraoxonasel activity is a risk for atherosclerosis in patients with systemic lupus erythematosus," Annals of the New York Academy of Sciences, vol. 1108, pp. 83-91, 2007.

[55] B. MacKness, M. I. MacKness, S. Arrol et al., "Serum paraoxonase (PON1) 55 and 192 polymorphism and paraoxonase activity and concentration in non-insulin dependent diabetes mellitus," Atherosclerosis, vol. 139, no. 2, pp. 341-349, 1998.

[56] M. Rosenblat, D. Draganov, C. E. Watson, C. L. Bisgaier, B. N. La Du, and M. Aviram, "Mouse macrophage paraoxonase 2 activity is increased whereas cellular paraoxonase 3 activity is decreased under oxidative stress," Arteriosclerosis, Thrombosis, and Vascular Biology, vol. 23, no. 3, pp. 468-474, 2003.

[57] S. T. Reddy, D. J. Wadleigh, V. Grijalva et al., "Human paraoxonase-3 is an HDL-associated enzyme with biological activity similar to paraoxonase-1 protein but is not regulated by oxidized lipids," Arteriosclerosis, Thrombosis, and Vascular Biology, vol. 21, no. 4, pp. 542-547, 2001.

[58] C. J. Ng, D. J. Wadleigh, A. Gangopadhyay et al., "Paraoxonase2 is a ubiquitously expressed protein with antioxidant properties and is capable of preventing cell-mediated oxidative modification of low density lipoprotein," Journal of Biological Chemistry, vol. 276, no. 48, pp. 44444-44449, 2001.

[59] H. Mochizuki, S. W. Scherer, T. Xi et al., "Human PON2 gene at 7q21.3: cloning, multiple mRNA forms, and missense polymorphisms in the coding sequence," Gene, vol. 213, no. 1-2, pp. 149-157, 1998.

[60] Q. Chen, S. E. Reis, C. M. Kammerer et al., "Association between the severity of angiographic coronary artery disease and paraoxonase gene polymorphisms in the National Heart, Lung, and Blood Institute-sponsored Women's Ischemia Syndrome Evaluation (WISE) study," American Journal of Human Genetics, vol. 72, no. 1, pp. 13-22, 2003.

[61] E. A. Ozer, A. Pezzulo, D. M. Shih et al., "Human and murine paraoxonase 1 are host modulators of Pseudomonas aeruginosa quorum-sensing," FEMS Microbiology Letters, vol. 253, no. 1, pp. 29-37, 2005.

[62] D. A. Stoltz, E. A. Ozer, C. J. Ng et al., "Paraoxonase-2 deficiency enhances Pseudomonas aeruginosa quorum sensing in murine tracheal epithelia," American Journal of Physiology, vol. 292, no. 4, pp. L852-L860, 2007.

[63] D. A. Stoltz, E. A. Ozer, P. J. Taft et al., "Drosophila are protected from Pseudomonas aeruginosa lethality by transgenic expression of paraoxonase-1," Journal of Clinical Investigation, vol. 118, no. 9, pp. 3123-3131, 2008.

[64] J. Ronald, R. Rajagopalan, J. E. Ranchalis et al., "Analysis of recently identified dyslipidemia alleles reveals two loci that contribute to risk for carotid artery disease," Lipids in Health and Disease, vol. 8, article 52, 2009.

[65] J. K. Pritchard, M. Stephens, and P. Donnelly, "Inference of population structure using multilocus genotype data," Genetics, vol. 155, no. 2, pp. 945-959, 2000.

[66] V. H. Brophy, R. L. Jampsa, J. B. Clendenning, L. A. McKinstry, G. P. Jarvik, and C. E. Furlong, "Effects of 5' regulatory-region polymorphisms on paraoxonase-gene (PON1) expression," American Journal of Human Genetics, vol. 68, no. 6, pp. 14281436, 2001.

[67] C. S. Carlson, M. A. Eberle, M. J. Rieder, Q. Yi, L. Kruglyak, and D. A. Nickerson, "Selecting a Maximally Informative Set 
of Single-Nucleotide Polymorphisms for Association Analyses Using Linkage Disequilibrium," American Journal of Human Genetics, vol. 74, no. 1, pp. 106-120, 2004.

[68] C. Chelala, A. Khan, and N. R. Lemoine, "SNPnexus: a web database for functional annotation of newly discovered and public domain single nucleotide polymorphisms," Bioinformatics, vol. 25, no. 5, pp. 655-661, 2009.

[69] S. Deakin, I. Leviev, S. Guernier, and R. W. James, "Simvastatin modulates expression of the PON1 gene and increases serum paraoxonase: a role for sterol regulatory element-binding protein-2," Arteriosclerosis, Thrombosis, and Vascular Biology, vol. 23, no. 11, pp. 2083-2089, 2003.

[70] D. I. Draganov, J. F. Teiber, A. Speelman, Y. Osawa, R. Sunahara, and B. N. La Du, "Human paraoxonases (PON1, PON2, and PON3) are lactonases with overlapping and distinct substrate specificities," Journal of Lipid Research, vol. 46, no. 6, pp. 1239-1247, 2005.

[71] L. M. Tripi, S. Manzi, Q. Chen et al., "Relationship of serum paraoxonase 1 activity and paraoxonase 1 genotype to risk of systemic lupus erythematosus," Arthritis and Rheumatism, vol. 54, no. 6, pp. 1928-1939, 2006.

[72] B. V. Kural, C. Örem, H. A. Uydu, A. Alver, and A. Örem, "The effects of lipid-lowering therapy on paraoxonase activities and their relationship with the oxidant-antioxidant system in patients with dyslipidemia," Coronary Artery Disease, vol. 15, no. 5, pp. 277-283, 2004.

[73] G. P. Jarvik, N. T. Tsai, L. A. McKinstry et al., "Vitamin C and E intake is associated with increased paraoxonase activity," Arteriosclerosis, Thrombosis, and Vascular Biology, vol. 22, no. 8, pp. 1329-1333, 2002.

[74] P. Koncsos, I. Seres, M. Harangi et al., "Favorable effect of short-term lifestyle intervention on human paraoxonase-1 activity and adipokine levels in childhood obesity," Journal of the American College of Nutrition, vol. 30, no. 5, pp. 333-339, 2011.

[75] L. G. Costa, G. Giordano, and C. E. Furlong, "Pharmacological and dietary modulators of paraoxonase 1 (PON1) activity and expression: the hunt goes on," Biochemical Pharmacology, vol. 81, no. 3, pp. 337-344, 2011.

[76] S. Bhaskar, M. Ganesan, G. R. Chandak et al., "Association of PON1 and APOA5 gene polymorphisms in a cohort of indian patients having coronary artery disease with and without type 2 diabetes," Genetic Testing and Molecular Biomarkers, vol. 15, no. 7-8, pp. 507-512, 2011.

[77] C. E. Furlong, S. M. Suzuki, R. C. Stevens et al., "Human PON1, a biomarker of risk of disease and exposure," ChemicoBiological Interactions, vol. 187, no. 1-3, pp. 355-361, 2010.

[78] Z. She, H. Chen, Y. Yan, H. Li, and D. Liu, "The human paraoxonase gene cluster as a target in the treatment of atherosclerosis," Antioxidants \& Redox Signaling, vol. 16, no. 6, pp. 597-632, 2012.

[79] D. A. Winnier, D. L. Rainwater, S. A. Cole et al., "Multiple QTLs influence variation in paraoxonase 1 activity in Mexican Americans," Human Biology, vol. 78, no. 3, pp. 341-352, 2006.

[80] J. Khateeb, A. Gantman, A. J. Kreitenberg, M. Aviram, and B. Fuhrman, "Paraoxonase 1 (PON1) expression in hepatocytes is upregulated by pomegranate polyphenols: a role for PPAR- $\gamma$ pathway," Atherosclerosis, vol. 208, no. 1, pp. 119-125, 2010. 

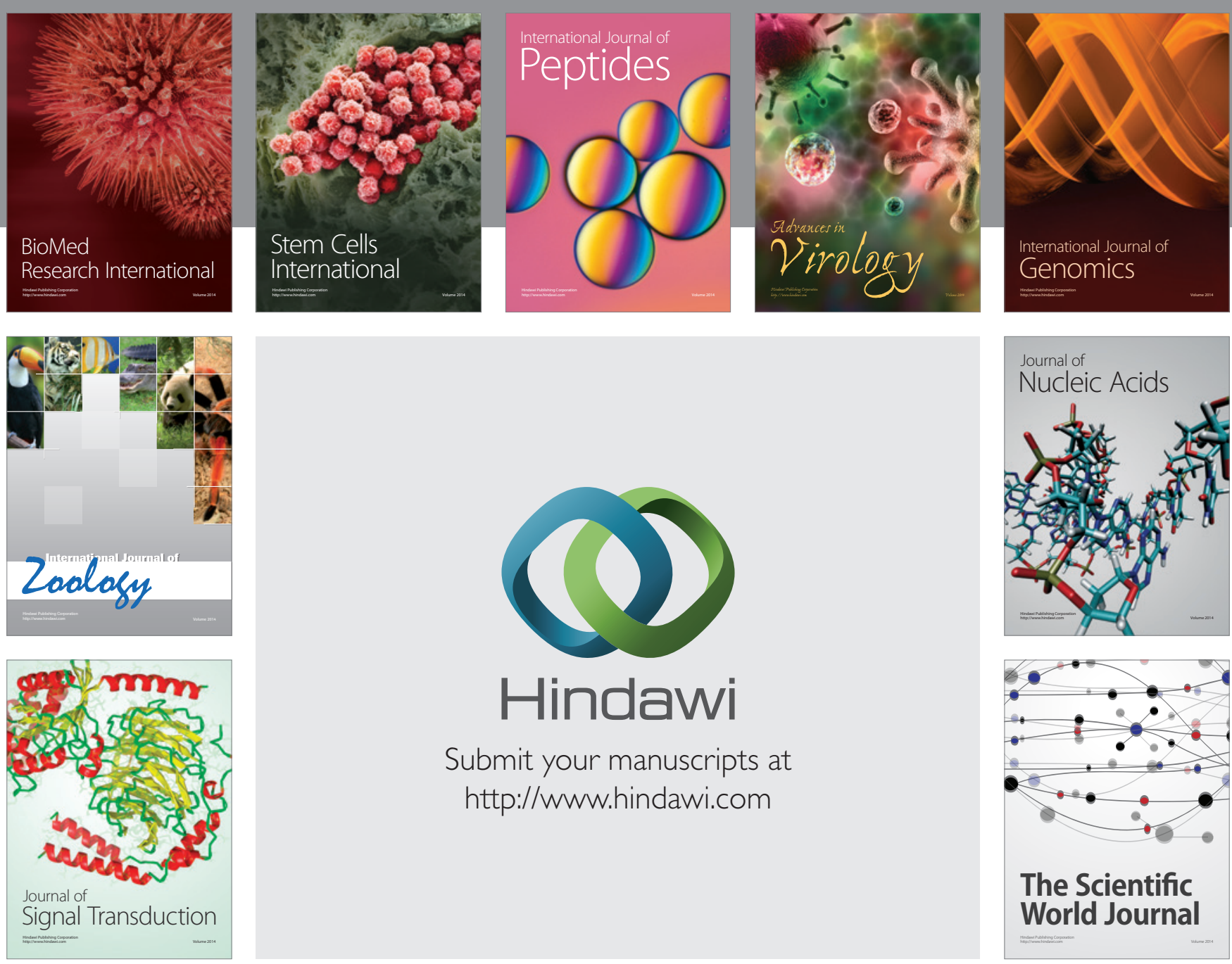

Submit your manuscripts at

http://www.hindawi.com
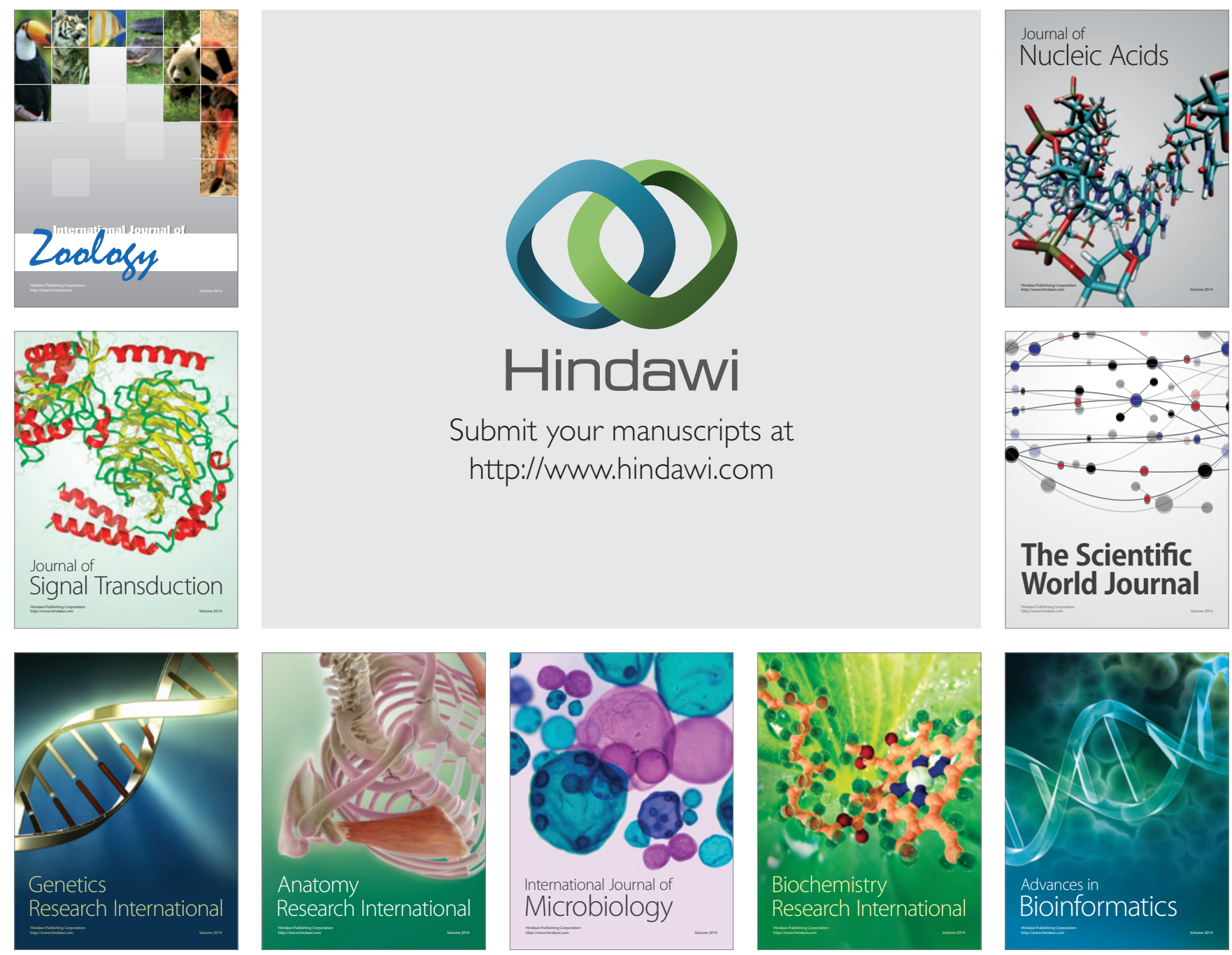

The Scientific World Journal
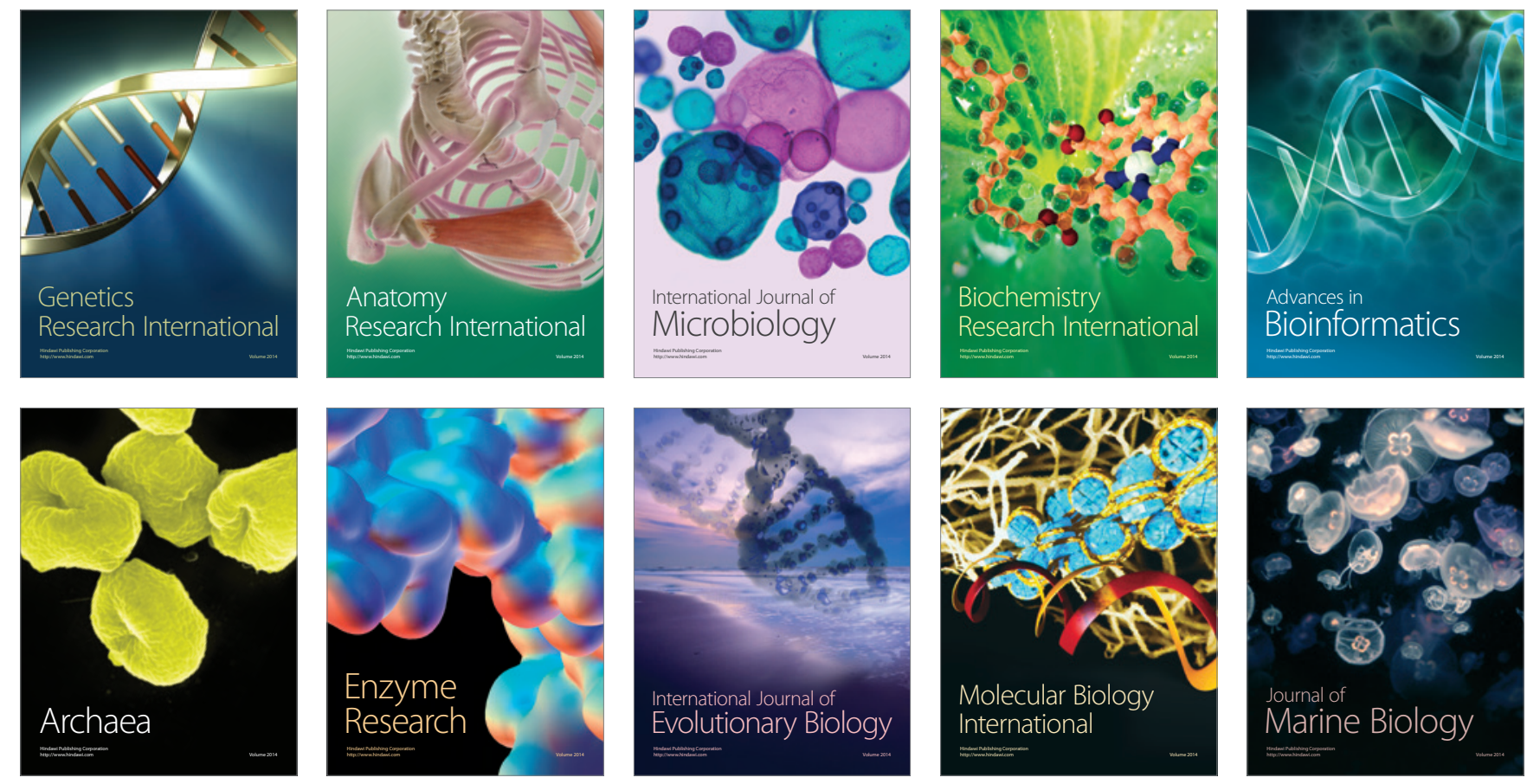